Annuaire du Collège de France 2017-2018

\title{
Les fixeurs au Moyen Âge
}

\section{Zrinka Stahuljak}

\section{(2) OpenEdition}

\section{Journals}

Édition électronique

URL : https://journals.openedition.org/annuaire-cdf/16498

DOI : 10.4000/annuaire-cdf.16498

ISBN : 978-2-7226-0572-5

ISSN : 2109-9227

Éditeur

Collège de France

Édition imprimée

Date de publication : 30 décembre 2020

Pagination : 718-720

ISBN : 978-2-7226-0516-9

ISSN : 0069-5580

Référence électronique

Zrinka Stahuljak, «Les fixeurs au Moyen Âge », L'annuaire du Collège de France [En ligne], 118|2020

mis en ligne le 01 avril 2021, consulté le 22 août 2022. URL : http://journals.openedition.org/annuairecdf/16498; DOI : https://doi.org/10.4000/annuaire-cdf.16498 


\title{
LES FIXEURS AU MOYEN ÂGE
}

\author{
Zrinka STAHULJAK \\ Professeure de littérature médiévale à l'université de Californie (États-Unis)
}

Mots-clés : Moyen Âge, fixeur

Cycle de quatre leçons données les $1^{\mathrm{er}}, 8,15$ et 22 juin 2018 (invitation sur proposition du Pr Patrick Boucheron), disponibles en audio sur le site internet du Collège de France : https://www.college-de-france.fr/site/patrick-boucheron/guestlecturer-2017-2018_1.htm.

Depuis l'engagement occidental en Afghanistan et en Iraq, le terme de fixeur (anglais, fixer) est devenu fréquent pour désigner, quasi exclusivement, des hommes qui rendent des services multiples aux journalistes et aux armées étrangères. Le fixeur est en réalité un très vieux terme journalistique qui désigne un homme à tout faire dans des situations de conflit : c'est celui qui est à la fois interprète, informateur, guide, médiateur, fournisseur, chauffeur, en bref, il fournit tout ce qu'il faut à un journaliste, voire à un militaire, pour survivre et travailler en terrain hostile. C'est un intermédiaire, un arrangeur qui possède de multiples savoirs et techniques. Son principal domaine d'action se situe donc dans des situations de conflit qui exigent une intervention bilingue, entre deux langues inintelligibles l'une pour l'autre.

Le fixeur n'est pas pour autant une profession ou un métier, mais une position très ancienne et qu'on trouve déjà au Moyen Âge. Le séminaire a donc proposé l'analyse du fixeur comme dispositif, résolument et médiéval et contemporain, dans une forme de passage intelligible entre les deux époques. De manière générale, le séminaire interrogeait notre rapport au monde: est-il immédiat ou passe-t-il par des intermédiaires?

La première leçon du séminaire, intitulée «Fixeurs, passeurs, lieux de passage : corps, textes et réseaux » a porté sur la définition des fixeurs, théorique et historique : les fixeurs existaient-ils au Moyen Âge ? Pourquoi préférer ce terme au passeur ou à l'interprète ? Pour comprendre l'absence des corps et des personnes dans l'étude du Moyen Âge, dont on a préféré majoritairement l'étude des textes, ont été abordés les clivages entre les disciplines de l'histoire (de la première modernité) et de la littérature (du Moyen Âge), et le statut canonique de «La tâche du traducteur » de Walter Benjamin et son rejet de la communication comme objectif de la traduction. À la place, quelle version de l'histoire du Moyen Âge peut-on écrire, quand le traducteur médiéval (travaillant sur l'ordre du prince, enfermé dans son étude) devient le fixeur (et sort dans le monde guidé par son propre désir et le marché) ? Et quand, par conséquent, la transmission des savoirs dépend de la communication rudimentaire et devient un réseau d'intermédiaires et l'impulsion à l'action ? L'exemple de Marco Polo en tant que fixeur a servi de fil conducteur dans cette première leçon qui se clôt sur son exemple, car la traduction latine du Devisement du monde de Marco Polo a incité Christophe Colomb à partir vers l'ouest en 1492. 
Dans les deux leçons suivantes, à travers les expériences historiques de vie et de mort des fixeurs au Proche- et Moyen-Orient ont été travaillés les discours d'éthique et d'économie du dispositif de fixeur, jusqu'à l'articulation de l'éthique sur l'économie.

Dans la deuxième leçon, «Stratégies et éthique (1) : fidélité », à partir des récits des missionnaires et des traités de croisade des XIII ${ }^{\mathrm{e}}$ et $\mathrm{XIV}^{\mathrm{e}}$ siècles, a été proposé le modèle composite du fixeur idéal : un homme né en Europe chrétienne, parlant les langues étrangères des nations infidèles ou schismatiques et connaissant leurs mœurs et coutumes, en pleine possession de ses connaissances et capacités, donc autonome dans sa capacité d'agir pour arriver à des fins de conversion et de domination du Proche-Orient par l'Occident chrétien. En même temps, un homme fidèle et dévoué, dans l'obéissance totale; le fixeur serait un moyen pur, sans volonté propre, se faisant l'intermédiaire invisible. Mais cette éthique qui s'enracinerait dans la fidélité des fixeurs s'inscrit dans les rapports de pouvoir et dans la finalité économique qui n'accordent pas la position de sujet au fixeur. Marco Polo, qui propose les langues comme marchandise et convertibilité, a de nouveau servi de fil conducteur à cette leçon et d'introduction à la suivante.

Dans la troisième leçon, «Éthique (2) et économie : don, courtoisie, pouvoir », a été reprise la notion du fixeur comme dispositif qui détermine notre relationalité au monde : puis-je être un sujet si je passe par un intermédiaire ? Par conséquent, puis-je accorder à un intermédiaire la position de sujet, puis-je lui laisser la vie ou dois-je lui donner la mort (le pouvoir souverain) ? C'était donc à travers la vie et la mort, du client comme de son fixeur, dans les récits de prédication et de pèlerinage au Proche-Orient, qu'ont été abordées les notions de don et de contrat (le travail des fixeurs relève-t-il d'une logique du marché, de l'économie d'échange ? Y a-t-il réciprocité, imputabilité, responsabilité envers l'autre ?), de courtoisie (que devient la «courtoisie» occidentale au Proche-Orient? Quel est le rapport entre la « courtoisie » et la vie sauve ?) et de pouvoir (avoir la puissance de faire multiplier les vies sauves au prix de la sienne sans avoir le pouvoir de garder la sienne : qui donc a le pouvoir?). S'il est certain que le fixeur s'avère être un personnage ambivalent (c'est celui qui assure la sauvegarde du client mais dont on a peur ; c'est celui qui a sa vie entre ses mains et peut jouer de ce pouvoir), comment cette ambivalence s'exprime-t-elle au niveau d'un réseau ou d'un état (le pouvoir souverain) dans le traitement des fixeurs ?

La dernière leçon du séminaire, «Le gouvernement des fixeurs : les Pays-Bas bourguignons », a été consacrée à la question, en apparence plus métaphorique, des «nations-fixeurs »: qu'advient-il quand un régime politique se veut être fixeur, comme la Bourgogne du $\mathrm{XV}^{\mathrm{e}}$ siècle ? Comment un état-fixeur devient un état des fixeurs ? L'empire, plutôt que l'état-nation, est-il l'entité politique des fixeurs ? Tout d'abord a été élargie la notion du réseau de fixeurs : l'ensemble de discours de la production culturelle - la culturalité - a été mise sur un pied d'égalité avec la gouvernementalité, puis traitée comme effective de gouvernementalité. Ainsi les bibliothèques bourguignonnes ont-elles été exploitées comme une archive de la production de l'avenir politique de la Bourgogne, une archive (historique) des (futurs) possibles. Elles ont ouvert la voie au grand empire transatlantique du $\mathrm{XVI}^{\mathrm{e}}$ siècle, celui de Charles V. Le séminaire s'est ainsi clos une dernière fois sur Marco Polo et l'exemple d'un réseau de culturalité. C'est un réseau, et non une seule œuvre, qui a changé la face du monde : Christophe Colomb lisait Marco Polo, Jean de Mandeville et les traités de croisade pour trouver la route de l'ouest vers 
Jérusalem. C'est donc l'horizon d'attente d'un réseau, des bibliothèques, qui permet l'écriture d'une nouvelle histoire connectée qui ne serait autre que l'histoire de la littérature connectée. 